NIST Technical Note 1971

\title{
Interference Tests at Room Temperature Applied to Deployed Low-Noise Receivers
}

Daniel G. Kuester

Duncan A. McGillivray

Adam Wunderlich

William F. Young

This publication is available free of charge from: https://doi.org/10.6028/NIST.TN.1971 
NIST Technical Note 1971

\title{
Interference Tests at Room Temperature Applied to Deployed Low-Noise Receivers
}

\author{
Daniel G. Kuester \\ Duncan A. McGillivray \\ William F. Young \\ RF Technology Division, Shared Spectrum Metrology Group \\ Communications Technology Laboratory \\ Adam Wunderlich \\ National Advanced Spectrum and Communications Test Network \\ Communications Technology Laboratory
}

This publication is available free of charge from:

https://doi.org/10.6028/NIST.TN.1971

October 2017

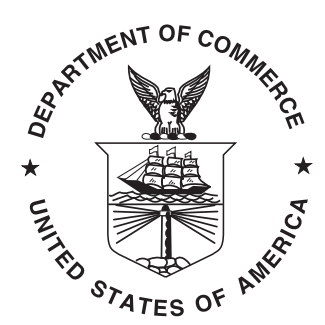

U.S. Department of Commerce

Wilbur L. Ross, Jr., Secretary

National Institute of Standards and Technology Walter Copan, NIST Director and Undersecretary of Commerce for Standards and Technology 
Certain commercial entities, equipment, or materials may be identified in this document in order to describe an experimental procedure or concept adequately. Such identification is not intended to imply recommendation or endorsement by the National Institute of Standards and Technology, nor is it intended to imply that the entities, materials, or equipment are necessarily the best available for the purpose.

National Institute of Standards and Technology Technical Note 1971 Natl. Inst. Stand. Technol. Tech. Note 1971, 23 pages (October 2017) CODEN: NTNOEF

This publication is available free of charge from: https://doi.org/10.6028/NIST.TN.1971 


\begin{abstract}
Wireless interference tests are often performed in shielded anechoic and semi-anechoic chambers near room temperature. The corresponding test condition experienced by a receiver device under test (DUT) is that the antenna noise temperature is equal to the physical temperature in the test chamber, barring electromagnetic interference (EMI).

This technical note considers a simple analytical model for estimating adjusted interference response from these room temperature tests for use in deployment antenna noise environments. The method applies to receivers that provide estimates of signal-to-noise ratio (SNR) that are approximately linear for low signal-to-interference-plus-noise ratio (SINR). The result of these estimates is most accurate when the total receiver system noise is not dominated by antenna noise temperature. Example applications include ground-based satellite receivers (for which antenna noise temperature is likely the averaged sky temperature) or cellular handsets (for which noise may originate primarily within the receiver electronics and antenna).

As a case study, we analyze test results published in NIST Technical Note 1952 [1]. The DUTs under study are global positioning system (GPS) L1 receivers exposed to antenna ("sky") noise temperatures of $90 \mathrm{~K}$ to $340 \mathrm{~K}$. For practical combinations of receiver noise figure and receive antenna efficiency performance, we develop an regression correction model that transforms interference power levels from the room temperature test environment into estimated equivalents in deployment. The regression is a function of antenna noise temperature, leaving receiver performance variability as a fit error. The worst-case error across the receiver performance parameter space was $\pm 1.6 \mathrm{~dB}$, which is tighter than the $\pm 2.4 \mathrm{~dB}$ uncertainty in the measurement of interference power level [1].
\end{abstract}




\section{Contents}

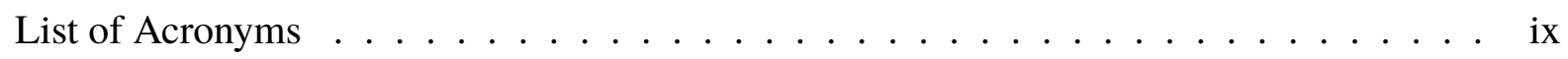

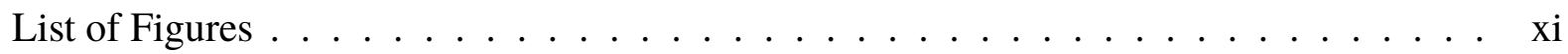

1 Introduction 1

1.1 Background . . . . . . . . . . . . . . . . . . 1

1.2 Measurement-Based Approaches to Receiver Noise Assessment . . . . . . . . . . 1

1.3 Numerical Simulation Approach based on Receiver Performance Constraints . . . . 2

2 Thermal Noise Model 5

2.1 Total Receiver System Noise . . . . . . . . . . . . . . . . . . . . . . . 5

2.2 Antenna and Brightness Temperatures . . . . . . . . . . . . . 6

2.3 Temperature, Power Spectral Density, and Band Noise Power . . . . . . . . . . 6

2.4 Measurement Link Assumptions . . . . . . . . . . . . . . . . . . . . 7

2.5 Derivation of Interference Adjustment Formula $\ldots \ldots \ldots \ldots$

2.5.1 Notation and Conventions . . . . . . . . . . . . . . 7

2.5 .2 SINR Isoresponse . . . . . . . . . . . . . . . . . . . . . 8

2.5.3 Limited Use of Receiver Self-Estimated SNR or $C / N_{0} \quad \ldots \ldots \ldots$

2.5.4 Interference Level Formula for Alternate Noise Conditions . . . . . . . . . 9

2.6 Model Limitations . . . . . . . . . . . . . . . . . . . . . . . . 10

3 Case Study: LTE Impacts on GPS 11

3.1 Noise Conditions in the Anechoic Test Environment . . . . . . . . . . . . . . . 11

3.2 GPS Receiver Performance Parameter Space . . . . . . . . . . . . . . . . . . . 12

3.3 Regression Model for Changes in System Temperature as a Function of Ambient Temperature . . . . . . . . . . . . . . . . . 12

3.4 Adjusted Interference Response as a Function of Ambient Temperature . . . . . . . 14

3.5 Adjusted Results . . . . . . . . . . . . . . . . . . . . . . 16

3.6 Results . . . . . . . . . . . . . . . . . . . . . . . 17

4 Conclusion $\quad 21$

$\begin{array}{lr}\text { References } & 23\end{array}$ 


\section{Acronyms}

DUT device under test

EMI electromagnetic interference

GPS global positioning system

LNA low-noise amplifier

LTE long-term evolution

OTA over-the-air

SINR signal-to-interference-plus-noise ratio

SNR signal-to-noise ratio

UL1 uplink band 1 (low) 


\section{List of Figures}

2.1 Modeled contributions to receiver system noise temperature $T_{\text {sys }}$. Ohmic losses in the antenna are modeled as an attenuator with attenuation $1 / \eta_{r}$ and physical temperature $T_{p}$. The receiver noise temperature $T_{\mathrm{rx}}$ is referred to the reference plane (indicated by the dot-

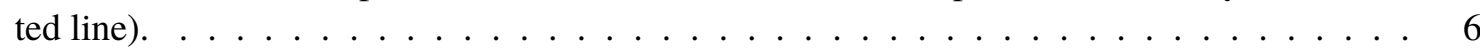

3.1 Change in relative system noise evaluated by (2.3) for many combinations of $T_{a}, \eta_{r}$, and noise figure. The central value has slope of $0.010 \mathrm{~dB} / \mathrm{K}$ and zero-intercept at $300 \mathrm{~K}$. . . . . 13

3.2 The shaded region represents the extreme range of possible errors in adjusted interference power level across the parameter space $\left(\eta_{r}, \mathrm{NF}\right.$, and $\left.T_{a}\right)$ relative to the center value fit of

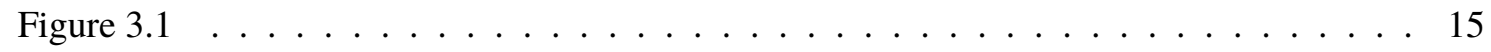

3.3 Corrected interference strength estimates as a function of interference strength of room

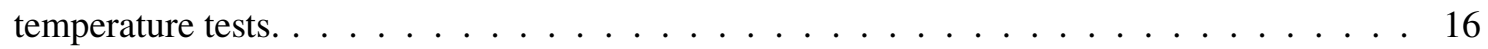

3.4 Estimates of LTE uplink band 1 (low) (UL1) impacts on the median $C / N_{0}$ reported by DUT 16 in [1] for antenna aperture temperatures $T_{a}=90 \mathrm{~K}, T_{a}=340 \mathrm{~K}$, each shown with

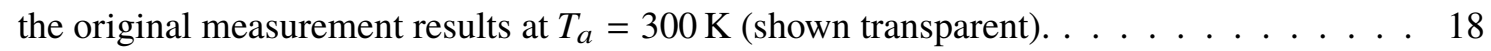

3.5 Estimates of LTE DL impacts on the median $C / N_{0}$ reported by DUT 7 in [1] for antenna aperture temperatures $T_{a}=90 \mathrm{~K}, T_{a}=340 \mathrm{~K}$, each shown with the original measurement

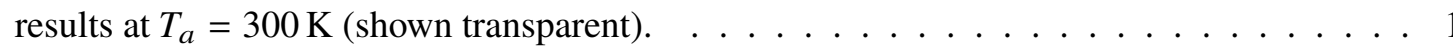




\section{Introduction}

\subsection{Background}

Recent work studied impacts of adjacent-band long-term evolution (LTE) cellular transmissions on GPS L1 receivers [1]. The results of the study are digital output responses of each GPS receiver for inputs that are mixtures of radiated GPS and LTE. These are the signal and interference strength, respectively. Application of these results requires SINR for the simplest flat fading channel model, and therefore noise performance inputs for each receiver.

The antenna noise temperature test condition in [1] was carefully controlled. The shielded anechoic and semi-anechoic test environments were chosen in part to reject outside radiated noise. The corresponding test condition for each GPS receiver is antenna noise temperature equal to physical room temperature (for highly emissive microwave absorber) - approximately $300 \mathrm{~K}$. Validation tests for EMI in the test zone ensured this condition.

A realistic environment will in general present a receiver with a noise level that differs from the test chamber. High-entropy interference sources or atmospheric effects may increase the noise level. Space-based or sky-facing receive antennas may present lower noise level. Quantitative assessment of total receive system noise is challenging in either case, because the total noise experienced by a receive system is a mixture of both the environment and receiver implementation. Radiated test methods that can separate these contributions are still subjects of active research. Recently proposed techniques [2] require the ability to disconnect the receive antenna from the receiver front-end, which is not practical for many integrated receivers.

When it is possible to assume that various receiver noise performance parameters lie within a constrained range, it may be significantly more practical to study the impacts of receiver noise by numerical simulation and not measurement. This technical note demonstrates this type of estimation with a case study on the anechoic test data in [1], in order to predict GPS "live sky" interference response based on anechoic chamber test behavior.

\subsection{Measurement-Based Approaches to Receiver Noise Assessment}

If rigorous measurement uncertainty is required in estimates of interference impacts, then the time, effort and expense of additional noise measurements are necessary. The practicality of this strategy depends largely on whether external antennas can be connected and disconnected for conducted measurements. 
A. For a receiver that can be disconnected from its antenna for testing, contributions from the receiver and antenna can be separated:

- Front-end receive electronics may be characterized by measuring the low-noise amplifier (LNA) noise figure (if it is separable), or with one-port methods like that in [2].

- Passive antenna receive noise performance may be characterized by radiation efficiency measurements

- Active antenna noise performance might be measured by $G / T$ [4]-[6] or plane-wavereferred noise [2].

B. For a receiver with an integrated antenna that cannot be disconnected for testing:

- Plane-wave-referred noise [2] of the receive antenna system, extended to be run in a thermal test chamber at various brightness temperatures.

Measurement and uncertainty analysis for these methods have only recently become active research topics. Tests undertaken by different parties may disagree substantially until mature test methods can be standardized.

The decision to undertake these measurements should not be taken lightly. Measurement of planewave-referred noise in [2] took approximately 8 hours for a single GPS receiver at a single ambient temperature. This could approximately double the time required to perform interference tests. The time and effort required for these tests needs to be weighed against the benefit of reduced (or at least more rigorously evaluated) uncertainty.

\subsection{Numerical Simulation Approach based on Receiver Performance Constraints}

Numerical simulation of total receive system noise requires assumed constraints on various receive system noise performance parameters.

A. One source of reasonable constraints is best practice for low-noise receiver design - connecting a noise-matched LNA close to the receive antenna output [3, p. 495]. This helps to minimize the input-referred noise temperature of the receiver electronics. In common radio frequency applications at carrier frequencies up to a few $\mathrm{GHz}$, the LNA noise figure lies between one and several $\mathrm{dB}$ (including conducted losses and potentially electrostatic discharge protection).

B. A reasonable range of receive antenna radiation efficiency performance values also constrain the potential thermal noise introduced inside the receive antenna itself. These are quantified by the antenna efficiency

These constraints, together with standard models for total receiver noise sources, permit computation of adjustments to the interference test conditions that estimate performance in realistic environments. 
The range of total system noise can be computed across this full receciver performance parameter space. If it is on the order of the measurement uncertainty in incident interference strength (or smaller), it is reasonable to find a formula that adjusts the interference test condition as a function of deployment antenna noise ("sky") temperature. The resulting noise-adjusted interference estimate may then be applied as part of a more realistic link model for system design or regulation. 


\section{Thermal Noise Model}

\subsection{Total Receiver System Noise}

We start by reviewing with the conventional model for receiver system noise temperature (e.g., [7]) and corresponding terminology [8]. Mismatch losses are not included but could be considered [9].

Contributions to system noise temperature are illustrated by Figure 2.1. The total system noise temperature, including each contribution, is

$$
T_{\mathrm{sys}}=T_{\mathrm{rx}}+\eta_{r} T_{a}+\left(1-\eta_{r}\right) T_{p}
$$

The parameters in each term are:

- The input-referred noise temperature of the receiver front-end is $T_{\mathrm{rx}}$. This is sometimes transformed to ts noise figure as $10 \log _{10}\left(1+T_{\mathrm{rx}} / T_{0}\right)$ (in $\left.\mathrm{dB}\right)$, where the reference temperature $T_{0}$ is usually defined as $290 \mathrm{~K}$;

- the antenna aperture temperature $T_{a}$ is determined by the brightness temperature distribution outside the antenna, including the sky, $T_{b}$; and,

- noise added due to ohmic losses inside the antenna are modeled at the physical temperature $T_{p}$.

The two sources of noise output by the antenna are weighted by the antenna efficiency, $\eta_{r}$. 


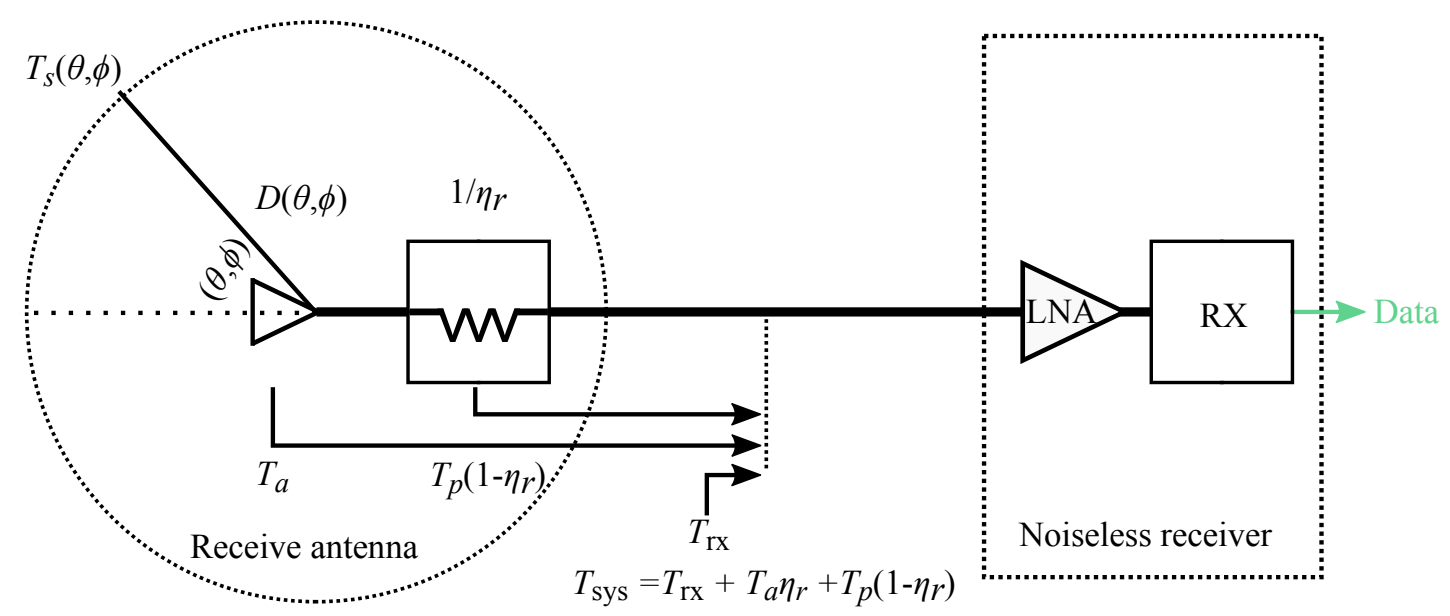

Figure 2.1: Modeled contributions to receiver system noise temperature $T_{\text {sys }}$. Ohmic losses in the antenna are modeled as an attenuator with attenuation $1 / \eta_{r}$ and physical temperature $T_{p}$. The receiver noise temperature $T_{\mathrm{rx}}$ is referred to the reference plane (indicated by the dotted line).

\subsection{Antenna and Brightness Temperatures}

The antenna aperture temperature $T_{a}$ is the average of the brightness temperature distribution $T_{b}$ across the radiansphere, weighted by antenna directivity $D$ :

$$
T_{a}=\frac{1}{4 \pi} \int_{0}^{2 \pi} \int_{0}^{\pi} D(\theta, \phi) T_{b}(\theta, \phi) \sin (\theta) d \theta d \phi .
$$

Evaluating the integral to estimate $T_{a}$ is discussed in other work, such as [10]. The remainder of this document works with $T_{a}$ as an input value, under the assumption it has already been measured or estimated.

\subsection{Temperature, Power Spectral Density, and Band Noise Power}

The input-referred system noise temperature, system noise-power, and system noise-density are related by the usual approximation for Johnson-Nyquist noise,

$$
N=B N_{0}=k B T_{\text {sys }}
$$

The system noise from thermal sources in a deployment scenario relative to that in the anechoic chamber at $T_{a} \rightarrow 300 \mathrm{~K}$ is

$$
\begin{aligned}
\frac{N}{\left.N\right|_{300 \mathrm{~K}}} & =\frac{T}{\left.T\right|_{300 \mathrm{~K}}} \\
& =\frac{\eta_{r} T_{\mathrm{a}}+\left(1-\eta_{r}\right) T_{p}+T_{\mathrm{rx}}}{\eta_{r}(300 \mathrm{~K})+\left(1-\eta_{r}\right) T_{p}+T_{\mathrm{rx}}}
\end{aligned}
$$


by substitution from (2.1). For the remainder of this technical note, the antenna aperture temperature $T_{a}$ left as a free variable. The physical temperature of the antenna and receiver front-end temperature are left constant, because

A. the physical antenna noise temperature does not change substantially across a reasonable range of terrestrial physical temperatures, and

B. the noise dependence on physical temperature was not measured or assessed in [1].

For B above, we emphasize that a practical receiver's noise performance does depend (and potentially significantly) on of the receiver's physical temperature [11]. If measurements across receiver physical temperature are available, this dependence can be included as well.

\subsection{Measurement Link Assumptions}

We make the following key assumptions as a basis for the rest of this note:

A. receiver response data in the presence of interference is a smooth function of SINR;

B. the receiver's self-estimate of $C / N_{0}$ is linear for low SINR;

C. the receiver's out-of-band rejection for interfering signal is linear with respect to power;

D. the physical temperature of the receiver, $T_{p}$, remains equal to $300 \mathrm{~K}$ as $T_{a}$ is changed; and

E. the receiver operates in a linear regime (the incident power is relatively weak after any RF filtering — this is necessary for assumption B).

Assumption A above is a key building block required to relate physical link models to DUT outputs. Achieving a smooth (not noisy) output for measurement may require averaging and substantial acquisition time. Confirmation of the assumption may require experimental or simulated validation (such as the study in Appendix B of [1]). Assumption B is supported by the simulation study and prior work [12], but only if the DUT implements one of the specific tested algorithms. Assumption $\mathrm{C}$ is reasonable (given Assumption B) if RF front-end filtering is passive and attenuates sufficient interference for the active components in the DUT to operate in a linear regime.

\subsection{Derivation of Interference Adjustment Formula}

\subsubsection{Notation and Conventions}

- Analysis is performed at the junction between the receive antenna and the receiver front-end.

- All quantities are computed in linear units. The conversion applied for $C / N_{0}$ quantities is:

$$
\begin{aligned}
C / N_{0} \text { in } \mathrm{dB}-\mathrm{Hz} & =10 \log _{10}\left(C / N_{0}\right) \\
C / N_{0} \text { in linear } \mathrm{Hz} & =10^{\left(C / N_{0} \text { in } \mathrm{dB}-\mathrm{Hz}\right) / 10} .
\end{aligned}
$$




\subsubsection{SINR Isoresponse}

In the presence of interference signals, the digitized baseband receive waveform in the DUT includes noise (with power $N$ or density $N_{0}$ ) and some total interference power $I$ that has leaked in from outside of the band (out-of-band).

By assumption A in Section 2.4, an equivalent receiver response may be achieved in the anechoic chamber and the realistic noise environment with slightly different interference power levels, $\left.I\right|_{300 \mathrm{~K}}$ and $I$ :

$$
\frac{C}{N+I}=\frac{C}{\left.N\right|_{300 \mathrm{~K}}+\left.I\right|_{300 \mathrm{~K}}} .
$$

The signal level condition under consideration is taken to be the same in either case, so

$$
N+I=\left.N\right|_{300 \mathrm{~K}}+\left.I\right|_{300 \mathrm{~K}}
$$

When $T_{a} \neq 300 \mathrm{~K}$, the interference power $I$ required to produce the same SINR (and receiver response) must change to reproduce the SINR at $300 \mathrm{~K}$.

\subsubsection{Limited Use of Receiver Self-Estimated SNR or $C / N_{0}$}

Data reported in [1] includes the self-reported estimates of $C / N_{0}$ output by each receiver. These included outputs reported with no interference, $\left.R_{0}\left(C / N_{0}\right)\right|_{300 \mathrm{~K}}$, and outputs reported with interference in the chamber, $\left.R_{I}\left(C / N_{0}\right)\right|_{300 \mathrm{~K}}$. Under assumption B in Section 2.4, and given that the carrier power levels are the same with and without interference:

$$
\left.\frac{C / N_{0}}{C /\left(N_{0}+I / B\right)}\right|_{300 \mathrm{~K}}=\left.\frac{N+I}{N}\right|_{300 \mathrm{~K}}=Y_{I} .
$$

The noise bandwidth $B$ cancels with $N_{0} \rightarrow N$. We normalize the total leaked interference power by $B$, producing an interference power density averaged across the equivalent bandwidth. We emphasize that this hides the expected (but unknown) frequency dependence in $N_{0}$, which may itself produce a substantial impact on receiver response compared to white noise. We rely on Assumption A in Section 2.4 to move forward, which can be interpreted as requiring that the DUT response to leaked interference is equivalent to that of in-band white additive noise.

The name $Y_{I}$ notation here is borrowed from the $Y$-factor noise measurement technique. In this adapted context, it is the degradation in SINR, or equivalently the relative increase in noise floor. It is computed in linear units as the ratio of SINR with and without interference. Its value should be greater than 1 . 
At relatively weak SINR, we use the following approximation:

$$
Y_{I} \approx \frac{\left.R_{0}\left(C / N_{0}\right)\right|_{300 \mathrm{~K}}}{\left.R_{I}\left(C / N_{0}\right)\right|_{300 \mathrm{~K}}} .
$$

This relies heavily on assumptions A and B in Section 2.4. It is difficult to bound the error in this approximation, because it depends on details of the receiver implementation. This error is likely to be largest when the linearity of $R\left(C / N_{0}\right)$ fails; this occurs for some estimators at larger $C / N_{0}$ as is shown in the simulation study of [1], Appendix B. The difficulty in bounding this error suggests that the use of this approximation is outside the traditional domain of metrology.

Solving for $\left.I\right|_{300 \mathrm{~K}}$ relates the interference and noise in terms of $Y_{I}$ as

$$
\left.I\right|_{300 \mathrm{~K}}=\left.N\right|_{300 \mathrm{~K}}\left(Y_{I}-1\right)
$$

\subsubsection{Interference Level Formula for Alternate Noise Conditions}

Substituting (2.11) into (2.8) leads to the solution for the received anechoic interference level in terms of noise and device output,

$$
N+I=\left(\left.N\right|_{300 \mathrm{~K}}\right)\left(1+Y_{I}-1\right)
$$

Therefore

$$
\frac{I}{\left.I\right|_{300 \mathrm{~K}}}=\frac{\left.N\right|_{300 \mathrm{~K}}}{\left.I\right|_{300 \mathrm{~K}}}\left(Y_{I}-\frac{N}{\left.N\right|_{300 \mathrm{~K}}}\right)
$$

and by again substituting (2.11),

$$
\frac{I}{\left.I\right|_{300 \mathrm{~K}}}=\frac{Y_{I}-\left(\frac{N}{\left.N\right|_{300 \mathrm{~K}}}\right)}{Y_{I}-1} .
$$

After conversion to decibel units, this is the estimated offset to interference power level (at $T_{a}=300 \mathrm{~K}$ ) that would achieve the same DUT response at antenna noise temperature $T_{a}$. The adjustment depends on $Y_{I}$ from baseline test data defined in (2.10) and the system noise temperature relative to the anechoic reference condition defined in (2.3).

The relative change in interference power applies to the received signal, as analyzed here, but also the EIIP [13] levels reported by [1], since the numerator and denominator are subject to the same frequency response. 


\subsection{Model Limitations}

The device self-estimated SNR or $C / N_{0}$ needs to be a smooth function of signal level plus noise. Otherwise, use of (2.10) to estimate $Y_{I}$ and adjusted interference levels by (2.12) risks measurement errors that are difficult to quantify. Large uncertainty or negative values of $I /\left.I\right|_{300 \mathrm{~K}}$ may result. Characterization of the amount of noise tolerable in the device output needs to be studied in order to bound this type of error. 


\section{Case Study: LTE Impacts on GPS}

We detail here an application of the previous chapter to data provided by [1]. The result is a procedure to estimate interference responses the GPS receiver DUTs across a range of deployment of antenna temperatures based on anechoic test data. The basis is a simple regression model for $N /\left.N\right|_{300 \mathrm{~K}}$, which applies across a parameter space of GPS receiver noise figure and antenna efficiency. Among all tested design parameter values and antenna aperture temperature as low as $90 \mathrm{~K}$, the most extreme regression errors in the resulting interference power estimate is $\pm 2.4 \mathrm{~dB}$, which is on the same order as the uncertainty of the incident interference strength in [1].

\subsection{Noise Conditions in the Anechoic Test Environment}

The antenna aperture temperature condition reported in the test results of [1] corresponds closely with

- the brightness temperature $T_{b}(\theta, \phi)$ equal to the $300 \mathrm{~K}$ physical temperature in the chamber across $\theta$ and $\phi$, and

- the physical receiver temperature in thermal equilibrium (with no mechanical thermal sink) with still ambient air at physical temperature $300 \mathrm{~K}$.

The noise characterization data in the report supports this interpretation.

- Noise levels output by the calibrated transmission system were specified in the report. Summary numbers are in 3.6.2.2 "Output Noise Floor," including validation data with analysis demonstrating negligible impact on DUT noise floor. This analysis is drawn from the more detailed validation test data presented in Appendix D.4, "Conducted Testbed Noise Floor Output."

- The anechoic chamber used in these tests has shielding effectiveness above $100 \mathrm{~dB}$, which is further enhanced by the installed RF absorber and ferrite tiles. The semi-anechoic chamber was shielded, though quantitative shielding effectiveness data are not available.

In both cases, no terrestrial services are licensed to radiate near the radio-navigation satellite services (RNSS) frequency band under study. This minimizes risk of any leakage of outside interference.

- Data for each DUT were collected without any other DUT inside the shielded chamber. The chamber itself was also checked for other additional sources of noise in the frequency bands under study. No measurable radiated noise was observed. 
We therefore conclude that each DUT antenna noise temperature during test was set by the physical temperature of the chamber absorber. This was equal to physical temperature, approximately $300 \mathrm{~K}$.

Note that if data cables or product implementation radiate noise that cause self-interference, system noise in the receiver noise floor may increase. Increased LTE power would be necessary to overcome the increased system noise in the GPS receiver. For any receivers in which this is the case, the model presented in this note will tend to overestimate impacts of LTE.

\subsection{GPS Receiver Performance Parameter Space}

The application of test data now leaves the regime of metrology and enters engineering estimation. We switch to use of the most extreme outcomes given a range of feasible inputs, because we do not have any reasonable basis to assign probability distributions to each performance parameter.

Evaluating (2.12) and its dependent parameters require data from several sources:

A. Test report result values from [1]:

- $T_{p}=300 \mathrm{~K}$ (E.2 in the report)

With respect to brightness temperature evaluated as uniformly $k T$, the variability in E.2 produces error below $0.05 \mathrm{~dB}$.

- The $R\left(C / N_{0}\right)$ are provided for each receiver under test (Chapter 6 in the report).

The adjusted interference levels need to be assessed for each tested receiver. For simplicity, we use the LTE power and $R\left(C / N_{0}\right)$ from the midpoint of the supplied uncertainty regions.

\section{B. Receiver noise performance values:}

Internal parameters in (2.3) are inside the "black box" receiver under test. We assess these across a range of reasonable design values. We computed $N /\left.N\right|_{300 \mathrm{~K}}$ across the following spans:

- $90 \mathrm{~K} \leq T_{a} \leq 340 \mathrm{~K}$

The proper value for this could be evaluated experimentally or analytically [10].

$-1 \mathrm{~dB} \leq$ Noise figure $(\mathrm{NF}) \leq 3 \mathrm{~dB}$, to compute receiver temperature as $T_{\mathrm{rx}}=(290 \mathrm{~K})\left(10^{\mathrm{NF} / 10}-1\right)$

- $50 \% \leq \eta_{r} \leq 100 \%$

\subsection{Regression Model for Changes in System Temperature as a Function of Ambient Tem- perature}

We computed $N /\left.N\right|_{300 \mathrm{~K}}$ with (2.4) across all combinations of range extrema in the parameter space. This resembles Monte Carlo analysis, though since we are only looking for extremes, it should be regarded as computing the range of possibilities within the parameter space, rather than 


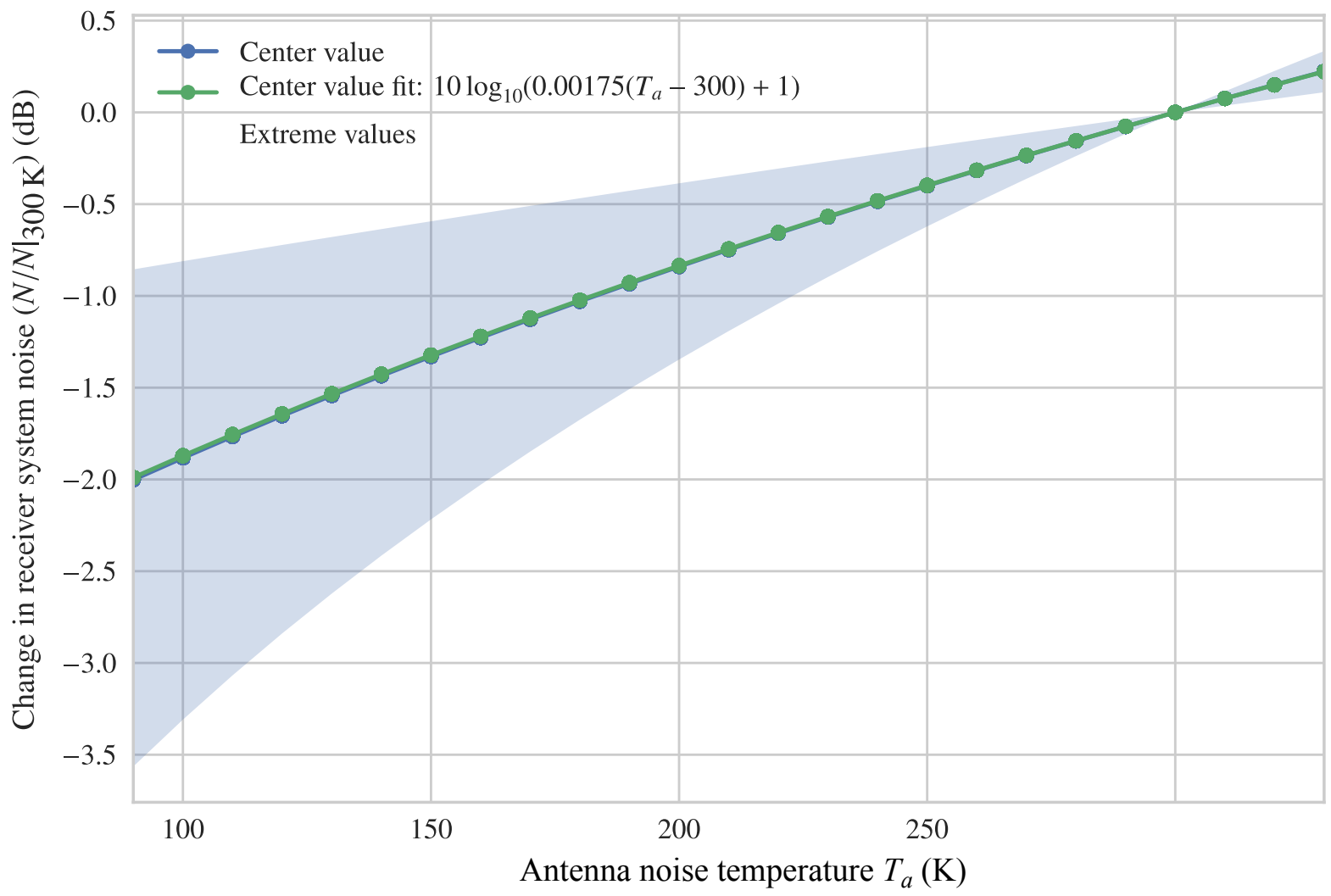

Figure 3.1: Change in relative system noise evaluated by (2.3) for many combinations of $T_{a}, \eta_{r}$, and noise figure. The central value has slope of $0.010 \mathrm{~dB} / \mathrm{K}$ and zero-intercept at $300 \mathrm{~K}$. 
a statistical result. The use of extrema is valid here because (2.4) includes no unsupported values in the given parameter space. The extremes are denoted by the region swept with antenna aperture temperature in Figure 3.1.

The mean value (in green) may be interpreted as the value for a "nominal" GPS receiver, and used as an approximate formula for the change in $C / N_{0}$. It is closely approximated by the following empirical equation:

$$
\frac{N}{\left.N\right|_{300 \mathrm{~K}}} \text { in } \mathrm{dB} \approx\left(T_{a}-300 \mathrm{~K}\right) \times 0.0105 \mathrm{~dB} / \mathrm{K} .
$$

Within the specified parameter space spanning $\left\{\eta_{r}, T_{a}, \mathrm{NF}\right\}$, the error between this approximation relative to the computed extrema (the envelope around the blue points) is within $\pm 1.6 \mathrm{~dB}$.

\subsection{Adjusted Interference Response as a Function of Ambient Temperature}

Correction factors to "live sky" antenna aperture temperature computed with (2.12) and (3.1) yield estimates of compensated interference power in a hypothetical "live sky" scenario. We sweep results across with integrated antenna temperature $T_{a}$. The $R\left(C / N_{0}\right)$ test data can then be referenced to the compensated interference power levels to obtain the equivalent impacts of LTE on GPS in a "live sky" deployment with an integrated antenna aperture temperature of $T_{a}$.

The compensated LTE power level for the horizontal axis is

$$
I \text { in } \mathrm{dBm}=\left.I\right|_{300 \mathrm{~K}} \text { in } \mathrm{dBm}+\left(\frac{I}{\left.I\right|_{300 \mathrm{~K}}}\right) \text { in } \mathrm{dBm} \text {, from (2.12). }
$$

To investigate the impacts of potential variability in GPS receiver noise performance, we computed the error in (3.2) resulting from use of the approximation (3.1) compared to that of the exact (2.3). In this computation exercise, the parameters are:

- $90 \mathrm{~K} \leq T_{a} \leq 340 \mathrm{~K}$

- $1 \mathrm{~dB} \leq$ Noise figure $(\mathrm{NF}) \leq 3 \mathrm{~dB}$, to compute receiver temperature as $T_{\mathrm{rx}}=(290 \mathrm{~K})\left(10^{\mathrm{NF} / 10}-1\right)$

- $50 \% \leq \eta_{r} \leq 100 \%$

- $1 \mathrm{~dB} \leq Y_{I} \leq 10 \mathrm{~dB}$

These form a 4-dimensional space of receiver performance parameters. We sample uniformly in each dimension and evaluate (3.2) for each combination.

The relative error in compensated $I$ is the difference (in $\mathrm{dB}$ ) between the approximated and exact $I$, shown in Figure 3.2. The error between the $T_{a}$ regression and the constrained space of "reasonable" receiver noise performance parameters is within $\pm 1.6 \mathrm{~dB}$. These bounds are tighter than the radiated uncertainty level of $\pm 2.4 \mathrm{~dB}$ from [1]. 


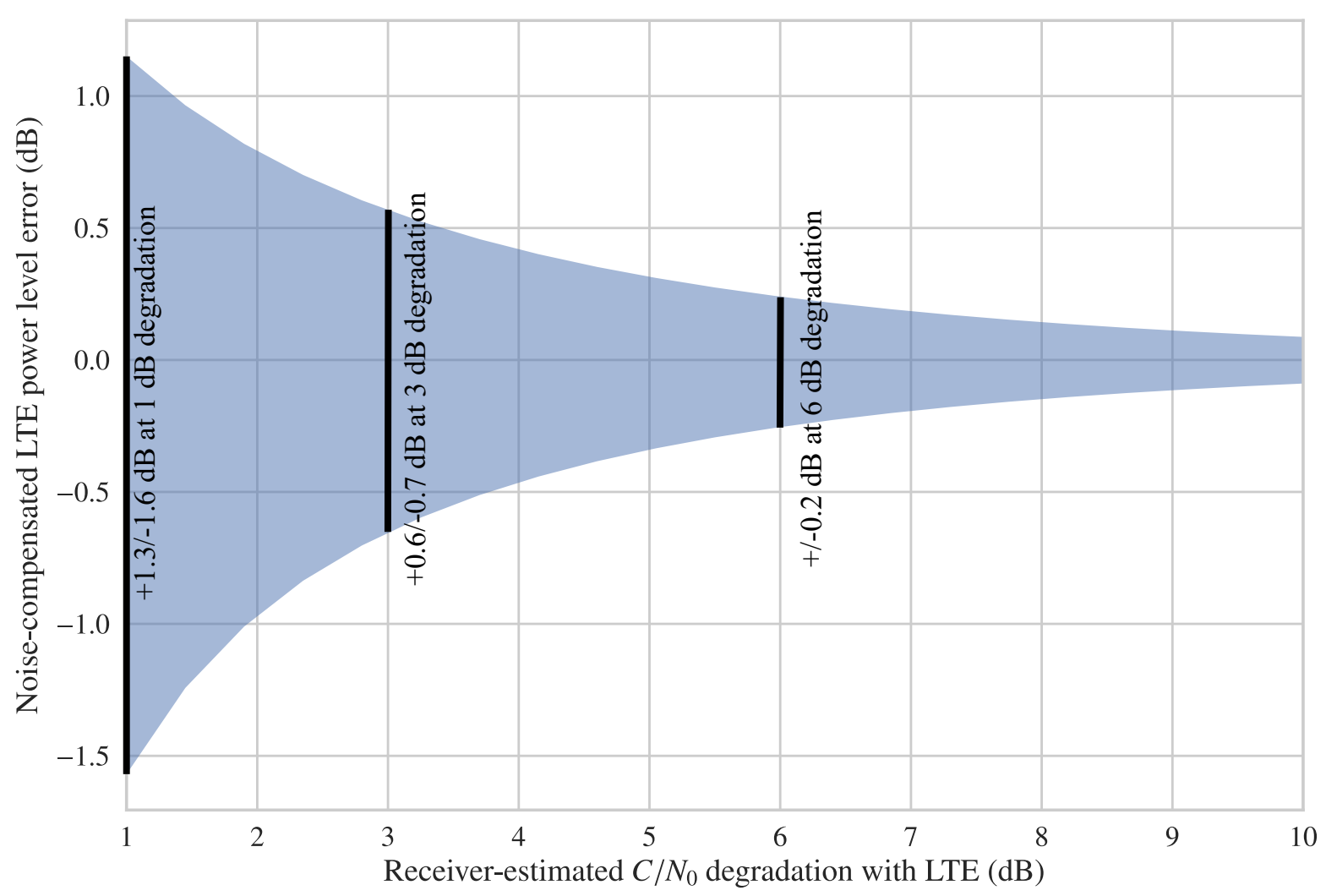

Figure 3.2: The shaded region represents the extreme range of possible errors in adjusted interference power level across the parameter space $\left(\eta_{r}, \mathrm{NF}\right.$, and $\left.T_{a}\right)$ relative to the center value fit of Figure 3.1 


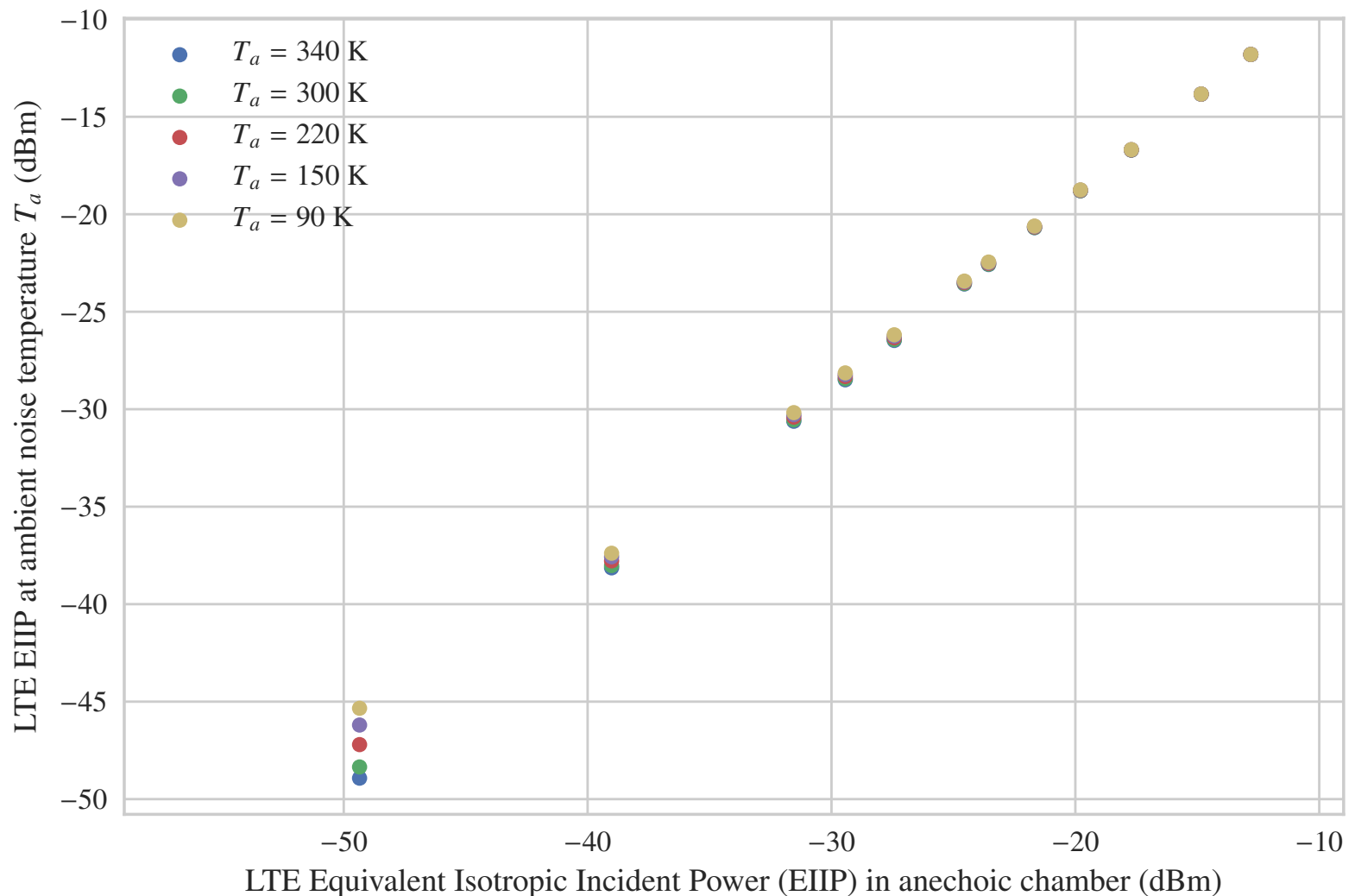

Figure 3.3: Corrected interference strength estimates as a function of interference strength of room temperature tests.

\subsection{Adjusted Results}

Consider as an example DUT 16 exposure to LTE uplink 1 (UL1), reported in [1][p. 379 Fig. F.26]. In order to satisfy the constraint of estimation based on low $C / N_{0}$, we used only the $C / N_{0}$ reported by the device for limited exposure for the GPS satellites at the weakest carrier power level. The corresponding satellites in this test case were PRN 15 and PRN 26, each at exposure level $-143.5 \mathrm{dBm}$ EIIP. For simplicity, we used the center of the uncertainty region for $R\left(C / N_{0}\right)$ and the LTE UL1 exposure level of the device-reported $C / N_{0}$ values for the two satellites.

Measuring or modeling brightness temperature to compute $T_{a}$ is beyond the scope of this work, so we chose a spread of example values between $90 \mathrm{~K}$ (a scenario that might imitate a carefully sky-focused antenna pattern at night) up to $340 \mathrm{~K}$ (an omni-directional antenna over a hot surface during the day).

The result is shown in Figure 3.3. The green dots represent $T_{a}=300 \mathrm{~K}$, the original data from anechoic testing. The other $T_{a}$ extremes show the most significant departure from the anechoic value at low interference power levels. The upper end of applied interference power is far greater than the thermal noise at all of these temperatures, so at these locations, the antenna aperture 
temperature makes little difference.

Another LTE UL1 power level tested in the report, $-60.2 \mathrm{dBm}$ EIIP, is not visible in the plot. This is because the $1 \mathrm{~dB}$ reporting resolution in DUT16 leads to results that are identical to the baseline data, producing $Y_{I}=1$ and therefore an indeterminate "divide by zero" from $Y_{I}-1$ in the denominator. Extension of any anechoic test data is less accurate when observed LTE impacts are small.

The curves fit to each $T_{a}$ point in Figure 3.3 also apply to other test types (other GPS signal scenarios or time-to-first-fix power tests) or other device outputs (3-D position error, number of satellites in view, or timing performance) for DUT 16 UL1. Tests of other DUTs or LTE scenarios (e.g., DL, UL2, or DL+UL1) would need separate curve fits based on the weakest satellite $C / N_{0}$ observed from the limited GPS scenarios.

\subsection{Results}

The net effect of the computed estimates of equivalent "live sky" interference response is to shift the location of the data points along the horizontal axis — the interference strength, LTE UL1 EIIP [2]. In order to illustrate the magnitude of this effect, results are shown for DUT 16 with LTE UL1 and DUT 7 with LTE DL in Figure 3.4 and Figure 3.5, respectively.

The full-width horizontal bars in these plots represent the $95 \%$ confidence region in the median output of the receiver at each satellite exposure level, without interference. The boxes with center values (denoted by circles) are the $95 \%$ confidence regions of the median outputs of the receiver. The width and height of these boxes represent the interference power and device output confidence region, respectively. These regions are taken directly from the result in [1]. Missing values denote that either the DUT did not respond at the indicated test condition (when transparent boxes are missing) or that the adjusted interference level estimate is non-physical (when solid boxes are missing).

The difference between the test data and the adjusted values demonstrate the behavior predicted by Figure 3.3: stronger interference levels tend to require smaller adjustments. 

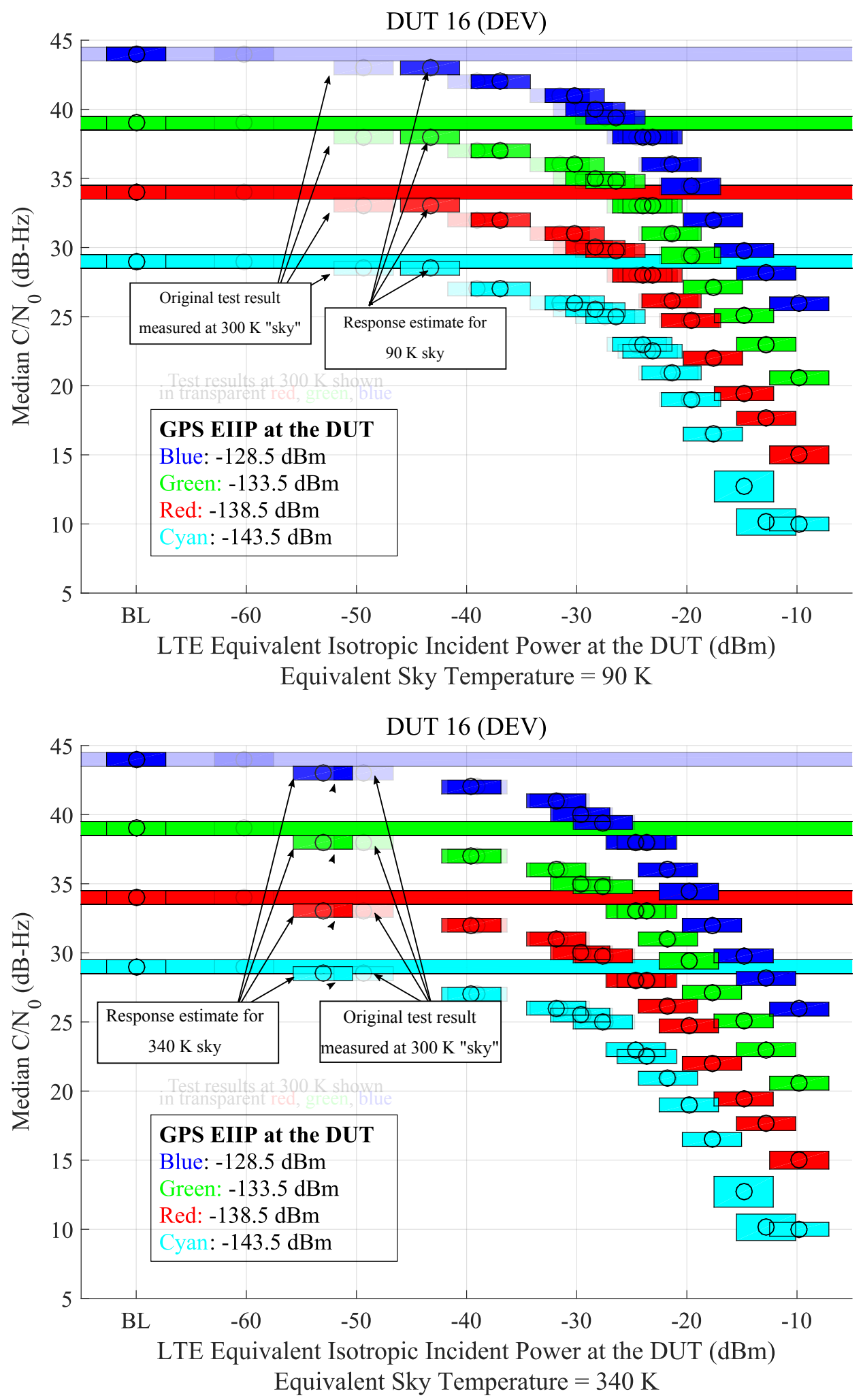

Figure 3.4: Estimates of LTE UL1 impacts on the median $C / N_{0}$ reported by DUT 16 in [1] for antenna aperture temperatures $T_{a}=90 \mathrm{~K}, T_{a}=340 \mathrm{~K}$, each shown with the original measurement results at $T_{a}=300 \mathrm{~K}$ (shown transparent). 


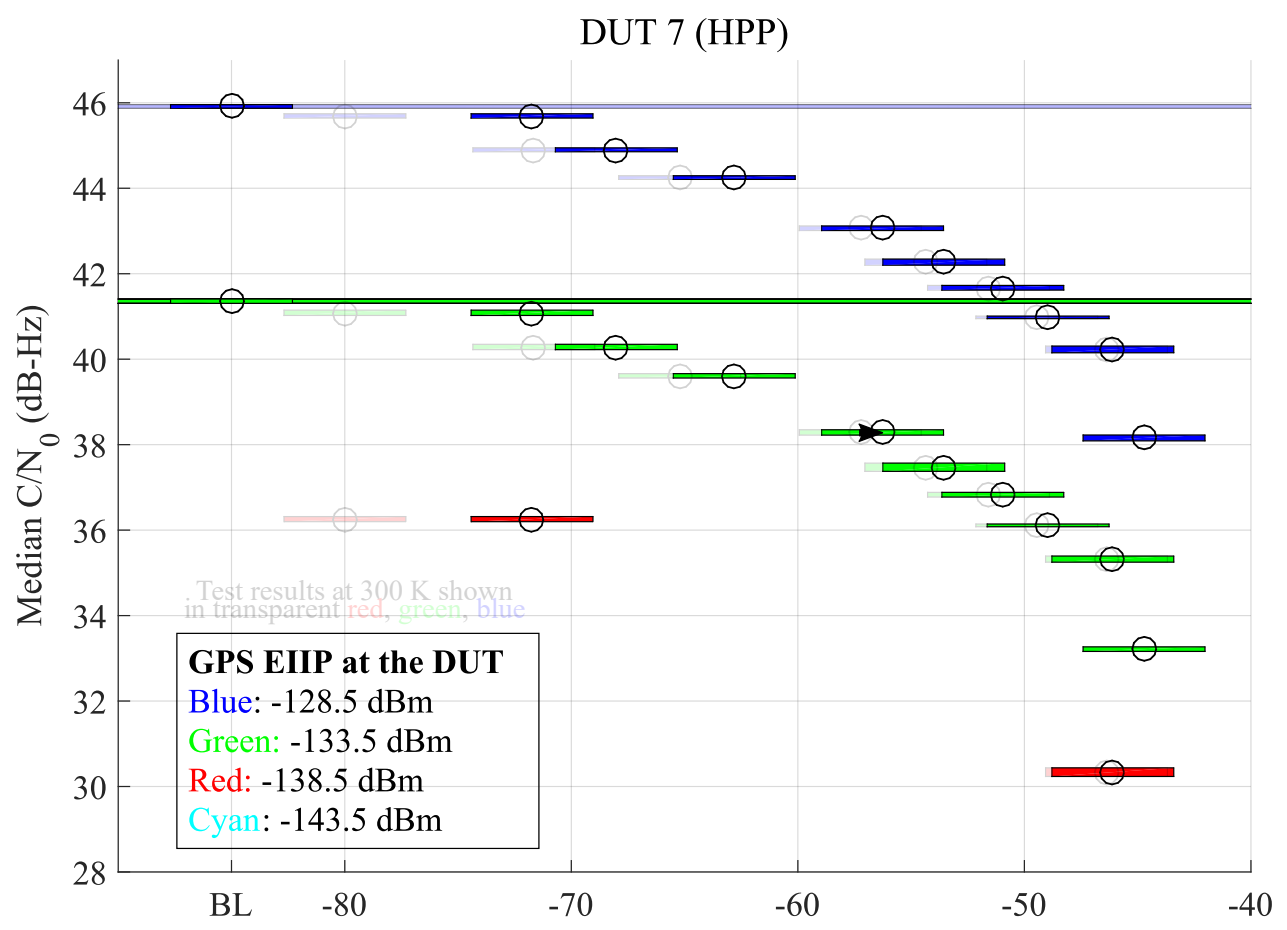

LTE Equivalent Isotropic Incident Power at the DUT (dBm)

Equivalent Sky Temperature $=90 \mathrm{~K}$

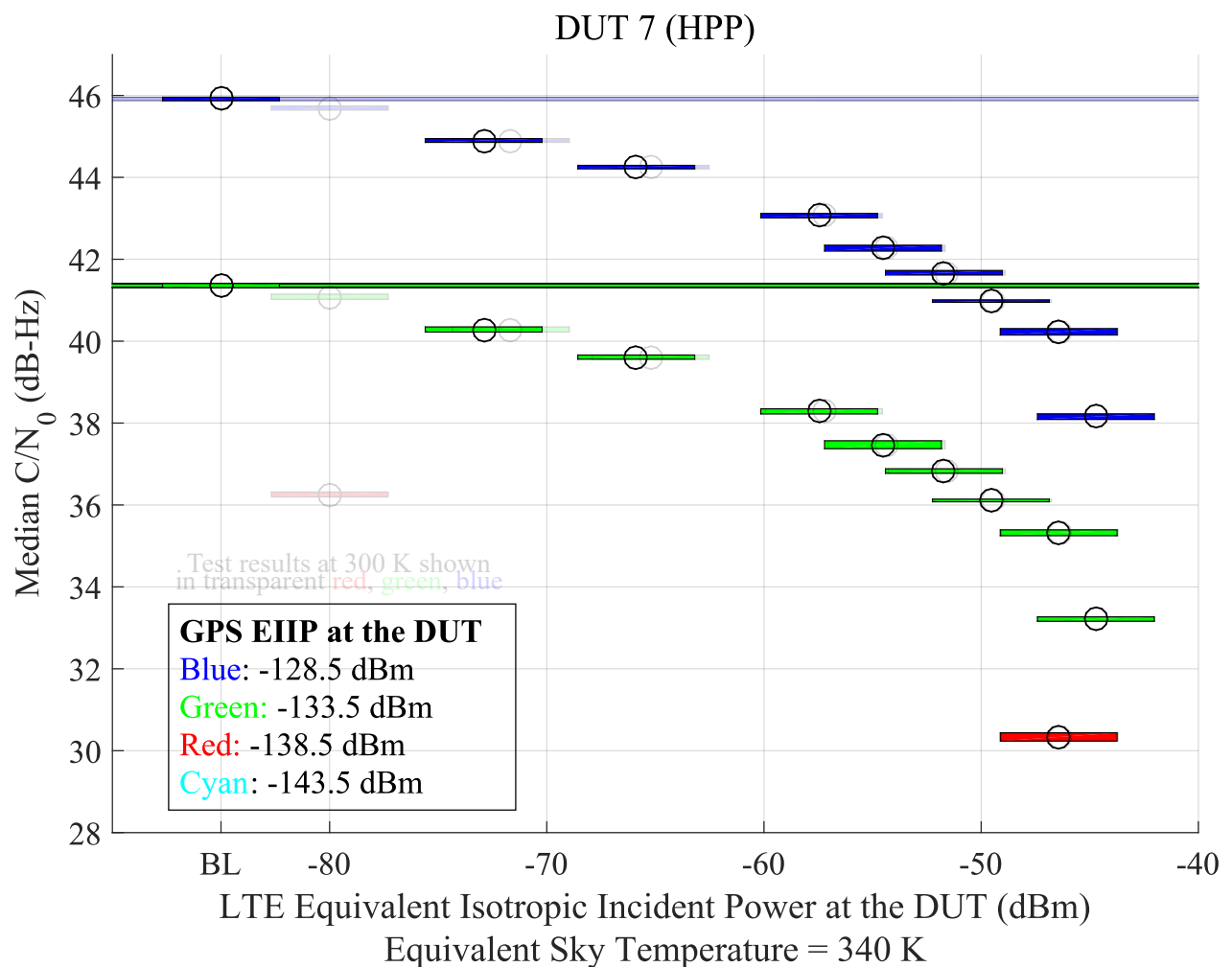

Figure 3.5: Estimates of LTE DL impacts on the median $C / N_{0}$ reported by DUT 7 in [1] for antenna aperture temperatures $T_{a}=90 \mathrm{~K}, T_{a}=340 \mathrm{~K}$, each shown with the original measurement results at $T_{a}=300 \mathrm{~K}$ (shown transparent). 


\section{Conclusion}

The analysis method discussed here applies to interference test results that did not include receiver noise characterization, requiring the assumption that the receiver self-estimate of SNR is accurate for low SNR levels. We relied on this assumption to perform a case study on the results of [1]. In this case, the variability in receiver noise performance that could be reasonably expected for GPS receivers yields worst-case error of $\pm 1.6 \mathrm{~dB}$ in adjusted interference power level for receiver SINR degradation as low as $1 \mathrm{~dB}$.

A stronger and more general approach based on measured noise levels would not depend on a receiver-provided SINR self-estimate (or its relative linearity). This may be possible in the near future for interference assessments performed in conjunction with measurements of receiver system noise. This type of noise measurement is a subject of ongoing research [2], and its application to over-the-air (OTA) interference measurements still requires significant development. 


\section{References}

[1] W. F. Young, S. Genco, et al., "LTE Impacts on GPS Final Report," NIST, Boulder, CO, Tech. Rep. 1952, Feb. 2017.

[2] D. G. Kuester, D. A. McGillivray, S. Genco, and D. Gu, "Black Box Measurement of System Noise in Integrated Wireless Receivers," in Proc. 2017 IEEE Conf. Antennas Propag. Wirel. Commun., Verona, Italy, 2017.

[3] D. M. Pozar, Microwave Engineering, 3rd. Hoboken, NJ: Wiley \& Sons, 2005.

[4] H. D. Griffiths and M. Libert, "Assessment of a technique for measurement of antenna G/T using solar noise," in Nov. Antenna Meas. Tech. IEE Colloq., 1994, pp. 6/1-6/6.

[5] P. Kolesnikoff, R. Pauley, and L. Albers, "G/T Measurement in an Anechoic Chamber," in Proc. Ant. Meas. Techn. Assoc., vol. 1, 2012, pp. 75-82.

[6] V. Rodriguez and C. Osborne, A Reduced Uncertainty Method for Gain over Temperature Measurements in an Anechoic Chamber. 2015.

[7] N. Razavi-Ghods, E. De, L. Acedo, A. El-Makadema, P. Alexander, and A. Brown, "Analysis of sky contributions to system temperature for low frequency SKA aperture array geometries," Exp Astron, vol. 33, pp. 141-155, 2012.

[8] J. P. Randa, J. Lahtinen, A. Camps, A. Gasiewski, M. Hallikainen, D. Le Vine, M. MartinNeira, P. Rosenkranz, C. S. Ruf, and N. Skou, "Recommended Terminology for Microwave Radiometry," Tech. Rep. Technical Note 1551, Aug. 2008.

[9] S. R. Best, "Realized Noise Figure of the General Receiving Antenna," IEEE Antennas Wirel. Propag. Lett., vol. 12, pp. 702-705, 2013.

[10] G. C. Medellin, “Antenna Noise Temperature Calculation,” Tech. Rep. Memo 95, 2007.

[11] J. Randa, J. Dunsmore, D. Gu, K. Wong, D. K. Walker, and R. D. Pollard, "Verification of noise-parameter measurements and uncertainties," IEEE Trans. Instrum. Meas., vol. 60, no. 11, pp. 3685-3693, 2011.

[12] E. Falletti, M. Pini, and L. L. Presti, "Low Complexity Carrier-to-Noise Ratio Estimators for GNSS Digital Receivers," IEEE Trans. Aerosp. Electron. Syst., vol. 47, no. 1, pp. 420-437, Jan. 2011.

[13] D. G. Kuester, D. A. McGillivray, J. Ladbury, A. Wunderlich, A. Feldman, W. F. Young, and S. Genco, "Equivalent Isotropic Response as a Surrogate for Incident Field Strength," in Proc. 2017 IEEE Int. Symp. Antennas Propag., San Diego, CA, 2017. 